\title{
SERGE PERRINE \\ Sur des équations diophantiennes généralisant celle de Markoff
}

\author{
Annales de la faculté des sciences de Toulouse $6^{e}$ série, tome $6, \mathrm{n}^{\circ} 1$ \\ (1997), p. 127-141 \\ <http://www.numdam.org/item?id=AFST_1997_6_6_1_127_0>
}

(C) Université Paul Sabatier, 1997, tous droits réservés.

L'accès aux archives de la revue «Annales de la faculté des sciences de Toulouse » (http://picard.ups-tlse.fr/ annales/) implique l'accord avec les conditions générales d'utilisation (http://www.numdam.org/conditions). Toute utilisation commerciale ou impression systématique est constitutive d'une infraction pénale. Toute copie ou impression de ce fichier doit contenir la présente mention de copyright.

\section{Numdam}

Article numérisé dans le cadre du programme Numérisation de documents anciens mathématiques http://www.numdam.org/ 


\title{
Sur des équations diophantiennes généralisant celle de Markoff $^{(*)}$
}

\author{
Serge Perrine ${ }^{(1)}$
}

RÉSUMÉ. - L'article examine les équations diophantiennes

$$
x^{2}+y^{2}+z^{2}=L x y z-u x \quad \text { (où } L \in \mathbb{N} \backslash\{0,1\} \text { et } u \in \mathbb{N} \text { ). }
$$

Ces équations généralisent celle de la théorie de Markoff. On donne toutes les solutions telles que $x, y, z$ sont des entiers strictement positifs lorsqu'elles existent. On montre qu'elles s'organisent alors en un nombre fini d'arbres. On donne une méthode pour énumérer toutes les équations diophantiennes de ce type ayant des solutions. Quelques compléments sur les points entiers de certaines surfaces cubiques sont donnés.

AbstraCt. - The article studies the diophantine equations

$$
x^{2}+y^{2}+z^{2}=L x y z-u x \quad(\text { where } L \in \mathbb{N} \backslash\{0,1\} \text { and } u \in \mathbb{N}) \text {. }
$$

These equations generalize the one encountered in the Markoff Theory. All the solutions such that $x, y, z$ are strictly positive integers when existing are given. It is shown that they can be organized in a finite number of trees. All such diophantine equations having solutions are given. Complements about entire points of some cubic surfaces are given.

\section{Introduction}

La théorie de Markoff classique consiste en l'étude de l'équation diophantienne :

$$
x^{2}+y^{2}+z^{2}=3 x y z \quad(x, y, z \text { entiers positifs }) .
$$

On sait (voir par exemple [1] ou [2]) que cette équation possède une infinité de solutions en nombres entiers strictement positifs. Elles sont données (à

(*) Reçu le 14 juin 1994

(1) Institut Supérieur de Génie Mécanique et Productique, Université de Metz, Ile-duSaulcy, F-57045 Metz Cedex 1 
une permutation près de $x, y$ et $z$ ) à partir de la solution fondamentale $(1,1,1)$ par une construction arborescente (fig. 1 ).

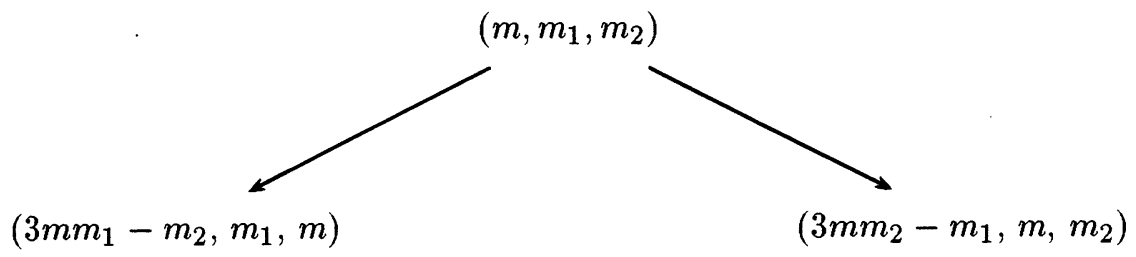

Fig. 1

Différentes généralisations de cette théorie ont été recherchées [3, pp. 106110 et $292-300]$. On a par exemple considéré les équations diophantiennes :

$$
\begin{aligned}
& x^{2}+y^{2}+z^{2}-L x y z=b \quad \text { (où } L \in \mathbb{N} \backslash\{0\} \text { et } b \in \mathbb{Z} \text { ) } \\
& x_{1}^{2}+x_{2}^{2}+\cdots+x_{n}^{2}-x_{0} x_{1} x_{2} \cdots x_{n}=0 .
\end{aligned}
$$

L'équation (3) a été complètement étudiée par A. Hurwitz ([4], [5] ou [15], [18]).

Elle recouvre la précédente si $b<0$ en posant $n=3-b, x_{0}=L, x=x_{1}$, $y=x_{2}, z=x_{3}$ et $\forall i=4 \cdots n, x_{i}=1$.

L'équation de Hurwitz (3) a donné lieu à des développements concernant les équations diophantiennes asymétriques ([6], [7]).

On a également étudié (cf. [8]) l'équation diophantienne :

$$
x^{2}-y^{2}+z^{2}=L x y z .
$$

Elle possède comme l'équation de Markoff (1) une infinité de solutions entières positives organisables en un arbre [8]. Ceci peut aussi se déduire des résultats de Silverman [9].

Mais ce n'est que très récemment ([10], [11]) qu'est apparue la possibilité de résoudre par une construction arborescente comparable des équations diophantiennes asymétriques qui généralisent naturellement l'équation de Markoff (1), telles que les suivantes :

$$
\begin{aligned}
& x^{2}+y^{2}+z^{2}=4 x y z-u x \quad(u \in \mathbb{N} \backslash\{0\}) \\
& x^{2}+y^{2}+z^{2}=L x y z-a_{12} x y-a_{23} y z-a_{31} x z-u_{1} y-u_{2} y-u_{3} z
\end{aligned}
$$

où $a_{12}, a_{23}, a_{31}, u_{1} u_{2}$ et $u_{3}$ sont des entiers tels que :

$$
L=3+a_{12}+a_{23}+a_{31}+u_{1}+u_{2}+u_{3} .
$$


Pour l'équation (5), T. W. Cusick [12] a complété les résultats disponibles en [11]. Il a montré que, dans certains cas, elle n'a pas de solution $(u=3$ ou $u=4)$.

Cette situation pour (5) est donc comparable à celle rencontrée par Frobenius [13] pour l'équation :

$$
x^{2}+y^{2}+z^{2}=L x y z, \quad L \in \mathbb{N} \backslash\{0,1,2,3\} .
$$

(Il s'agit de l'équation (2) avec $b=0$. Elle n'a pas de solution.)

Cusik a également vérifié que lorsque l'équation (5) a des solutions positives, elles s'organisent en un arbre infini $(u=1, u=2$ ou $u=5)$ ou en plusieurs arbres infinis $(u=10)$.

L'objet du présent article est de montrer que ces résultats de Cusick s'étendent à une classe plus vaste d'équations. Ces dernières équations ne rentrent d'ailleurs pas toutes dans la classe $\left(5^{\prime}\right)$ mentionnée par Manin [10, théorème 7.5.8] et étudiée dans [17]. Mais elles conduisent à des résultats comparables explicités dans la suite.

\section{Résultats}

On note dans la suite $\mathbb{N}^{*}=\mathbb{N} \backslash\{0\}$ ensemble des nombres entiers strictement positifs.

On considère l'équation diophantienne :

$$
x^{2}+y^{2}+z^{2}=L x y z-u x
$$

où $u \in \mathbb{N}^{*}$ et $L \in \mathbb{N}^{*}(L \geq 2)$.

On s'intéresse ici aux solutions $(x, y, z)$ telles que $x, y, z$ soient des éléments de $\mathbb{N}^{*}$.

Après avoir établi l'existence d'une infinité de solutions à partir de l'une d'elles, si elle existe, on systématise la construction de nouvelles solutions. On introduit la notion de solution fondamentale. Toute solution fondamentale est la racine d'un arbre de solutions dont on fournit le mode de construction. Ainsi, à partir d'une solution $s=(x, y, z)$, on en construit trois nouvelles :

$$
\begin{aligned}
\phi_{1}(s)=\left(x^{\prime}, y, z\right), \quad \phi_{2}(s) & =\left(x, y^{\prime}, z\right), \quad \phi_{3}(s)=\left(x, y, z^{\prime}\right) \\
- & 129-
\end{aligned}
$$




\section{Serge Perrine}

où

$$
x^{\prime}=L y z-x-u, \quad y^{\prime}=L x z-y, \quad z^{\prime}=L x y-z
$$

Elles sont à termes strictement positifs et sont dites voisines de la solution $(x, y, z)=s$.

Elles permettent à leur tour de construire de nouvelles solutions, et ainsi de suite par récurrence.

Les trois transformations $\phi_{1}, \phi_{2}, \phi_{3}$ correspondent géométriquement à des automorphismes de la surface réelle d'équation affine (7). Elles engendrent un groupe $G$ qui permet de décrire toutes les solutions de l'équation diophantienne (7) par action, le plus souvent transitive, de ce groupe.

Pour préciser les choses, on adapte comme le fait Cusick [12] la présentation de la théorie de Markoff faite par Cassels (cf. [1]).

Les solutions sont classées en considérant les quatre nombres :

$$
\begin{aligned}
m & =\max (x, y, z) \\
m_{x}^{\prime} & =\max \left(x^{\prime}, y, z\right) \\
m_{y}^{\prime} & =\max \left(x, y^{\prime}, z\right) \\
m_{z}^{\prime} & =\max \left(x, y, z^{\prime}\right) .
\end{aligned}
$$

Une solution $(x, y, z)$ est dite non fondamentale si et seulement si l'un des nombres $m_{x}^{\prime}, m_{y}^{\prime}$ et $m_{z}^{\prime}$ est strictement plus petit que $m$.

Elle est dite fondamentale dans le cas contraire.

On s'assure que pour toute solution non fondamentale $(x, y, z)$ deux des nombres $m_{x}^{\prime}, m_{y}^{\prime}$ et $m_{z}^{\prime}$ sont plus grands que $m$, le troisième étant plus petit que $m$.

Ceci permet de construire, à partir de toute solution non fondamentale deux branches dans l'arbre des solutions de l'équation considérée.

On a alors le résultat suivant.

ThÉoRÈme 1.- Si pour $u \in \mathbb{N}^{*}$ et $L \in \mathbb{N}^{*}(L \geq 2)$, l'équation diophantienne $x^{2}+y^{2}+z^{2}=L x y z-u x$ admet une solution $(x, y, z)$ en nombres entiers strictement positifs, alors elle en admet une infinité organisée en un ou plusieurs arbres ayant chacun pour racine une solution fondamentale de cette même équation. 
Ce résultat peut être présenté autrement en disant que le groupe d'automorphismes $G$ est un produit libre de trois copies de $\mathbb{Z} / 2 \mathbb{Z}$ agissant sur l'ensemble $S$ des solutions de (7) (voir [10] pour un résultat identique sur l'équation $\left.\left(5^{\prime}\right)\right)$.

Pour les solutions fondamentales, on montre alors le théorème 2 .

THÉORÈME 2. - L'équation diophantienne $x^{2}+y^{2}+z^{2}=L x y z-u x$, où $u \in \mathbb{N}^{*}$ et $L \in \mathbb{N}^{*}(L \geq 2)$ n'admet qu'un nombre fini de solutions fondamentales $(x, y, z)$ en nombres entiers strictement positifs. Elle n'admet donc qu'un nombre fini d'arbres de solutions.

Ce théorème signifie que l'ensemble $S$ est composé d'un nombre fini d'orbites pour l'action du groupe $G$. Chaque orbite est bijectivement associée à une solution fondamentale. L'ensemble des solutions fondamentales est fini. Il peut être vide comme pour l'équation (6). Il peut contenir un unique élément comme pour l'équation (1). Il peut aussi avoir plus de deux éléments, comme l'a montré Cusick [12] dans le cas de (5) avec $L=4$ et $u=10$ ou $u=25$.

Ceci conduit à rechercher comment identifier les solutions fondamentales, c'est-à-dire par ce qui précède, à déterminer complètement $S$.

On montre que les solutions fondamentales correspondent à la double décomposition d'un nombre entier positif en carrés.

Plus précisément, on a le résultat suivant.

THÉORÈME 3. - En énumérant tous les nombres entiers $T \in \mathbb{N}^{*}$ possédant deux décompositions en sommes de carrés de forme:

$$
T=(L x z)^{2}+u^{2}=(2 z)^{2}+(2 x+u)^{2}+v^{2}
$$

$o \grave{u}$

$$
L \in \mathbb{N}^{*} \quad(L \geq 2), \quad u \in \mathbb{N}^{*}, \quad v \in \mathbb{N}, \quad x \in \mathbb{N}^{*}, \quad z \in \mathbb{N}^{*}
$$

et en posant :

$$
y=\frac{L x z-v}{2} \quad(v \text { minimale }) .
$$

On détermine toutes les équations diophantiennes :

$$
x^{2}+y^{2}+z^{2}=L x y z-u x \quad \text { où } \quad L \in \mathbb{N}^{*}(L \geq 2) \text { et } u \in \mathbb{N}^{*} \text { donnés }
$$

possédant des solutions $(x, y, z) \in \mathbb{N}^{* 3}$ strictement positives, ainsi que toutes leurs solutions fondamentales. 
Inversement, toute équation diophantienne de forme (7) non ainsi obtenue à partir de deux décompositions en sommes de carrés d'un nombre $T \in \mathbb{N}^{*}$ ne possède pas de solution strictement positive.

Ce résultat donne un algorithme aisément automatisable qui permet d'identifier toutes les valeurs $L \in \mathbb{N}^{*}(L \geq 2)$ et $u \in \mathbb{N}^{*}$ telles que l'équation (7) ait des solutions en entiers strictement positifs, ainsi que les solutions fondamentales correspondantes.

$\grave{A}$ titre d'exemple, on donne maintenant toutes les possibilités ainsi trouvées pour $L=3$ avec $u \leq 20$, à la permutation près de $y$ et $z$ :

\begin{tabular}{|ll|ll|}
\hline$u$ & $(x, y, z)$ & $u$ & $(x, y, z)$ \\
\hline 0 & $(1,1,1)$ & 14 & $(2,2,6)$ \\
2 & $(2,1,3)$ & 15 & $(1,4,4)$ \\
3 & $(1,2,2)$ & & $(4,2,4)$ \\
4 & $(1,2,3)$ & 16 & $(2,3,3)$ \\
6 & $(2,2,2)$ & & $(13,1,18)$ \\
& $(5,1,7)$ & 18 & $(1,4,5)$ \\
8 & $(1,3,3)$ & & $(3,3,3)$ \\
10 & $(1,3,4)$ & 19 & $(1,4,6)$ \\
12 & $(2,2,4)$ & 20 & $(17,1,21)$ \\
& $(10,1,13)$ & & \\
\hline
\end{tabular}

\section{Démonstration des théorèmes 1 et 2}

On s'inspire ici directement de la méthode de Cusick [12] :

\subsection{Démonstration du théorème 1}

\subsubsection{Examen des différents cas possibles}

Les différents cas possibles se déduisent de la comparaison d'une solution et de ses solutions voisines.

Cas 1. - On suppose $x^{\prime}=x$. Alors $x=m_{x}^{\prime}$. Mais on a aussi, avec l'hypothèse faite sur $L$ :

$$
\begin{aligned}
& y^{\prime}=m_{y}^{\prime}>x=m>y \\
& z^{\prime}=m_{z}^{\prime}>x=m>z .
\end{aligned}
$$


De sorte que la solution $(x, y, z)$ est fondamentale. La configuration correspondante est donnée par la figure 2 .

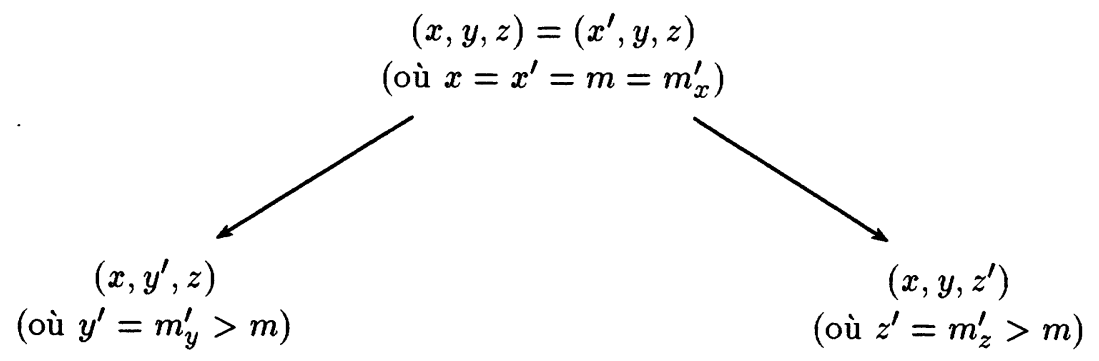

Fig. 2

Cette situation est possible par exemple dans le cas où $L=2, u=14$ avec $(x, y, z)=(5,4,3)$. Dans ce cas, le groupe $G$ n'agit pas transitivement sur l'ensemble $S$ des solutions de l'équation (7).

Cas 2. - On suppose $x^{\prime}>x$. Alors $x^{\prime}=m_{x}^{\prime}>m$.

Observant alors que si $(x, y, z)$ est solution de l'équation, $(x, z, y)$ est également une solution, on peut supposer que l'on a :

$$
y=\max (y, z) \geq z
$$

Alors $z^{\prime}>y$.

Cette inégalité permet de distinguer deux cas qui donnent tous deux $m_{z}^{\prime}>m$. Ce sont les suivants :

a) si $y \geq x$, alors $z^{\prime}=m_{z}^{\prime}>y=m \geq z$;

b) si $x>y$, alors $z^{\prime}=m_{z}^{\prime}>x=m>y \geq z$.

On considère alors les deux possibilités suivantes :

- si $(x, y, z)$ est une solution non fondamentale; on a nécessairement $m_{y}^{\prime}<m$,

- le cas 2 a) est possible avec :

$$
y=m>m_{y}^{\prime} \geq y^{\prime}
$$


la configuration correspondante est donnée par la figure 3 .

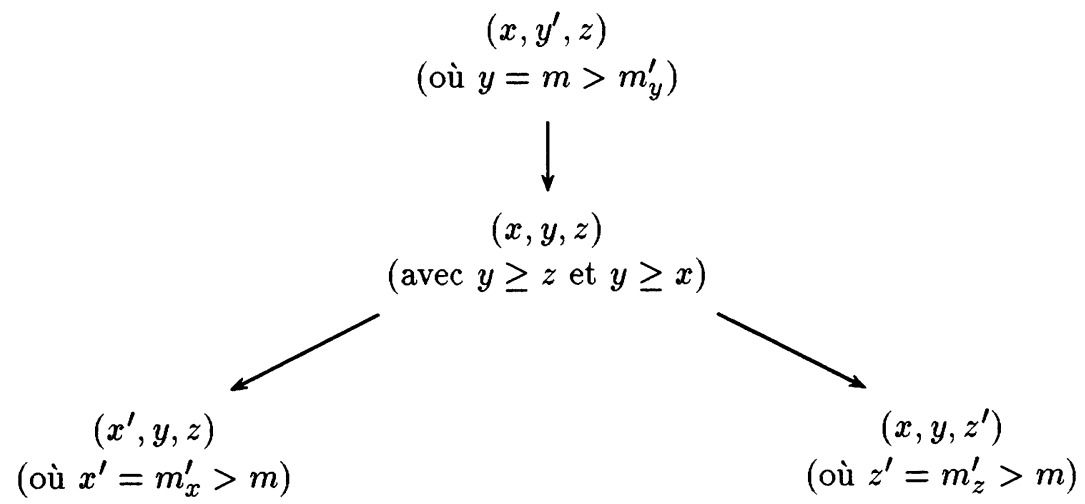

Fig. 3

- le cas 2 b) est impossible;

- si $(x, y, z)$ est une solution fondamentale; on a $m_{y}^{\prime} \geq m$,

- dans le cas 2 a), on a :

$$
\max (x, z)<y=m \leq m_{y}^{\prime}=\max \left(y^{\prime}, \max (x, z)\right)
$$

d'où aussi $y^{\prime}=m_{y}^{\prime}$;

- dans le cas $2 \mathrm{~b}$ ), on a, avec l'hypothèse sur $L, y^{\prime}=m_{y}^{\prime}$;

dans ces deux cas, la configuration correspondante est, si $y^{\prime}>y$, celle donnée par la figure 4 .

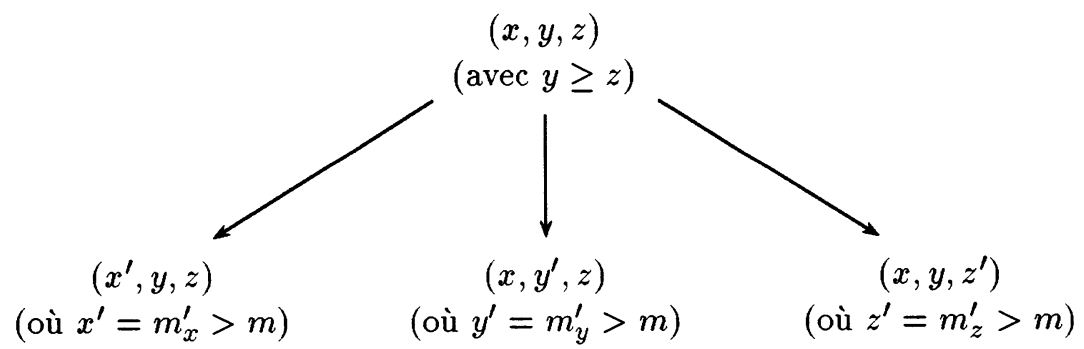

Fig. 4

Cette situation est possible par exemple dans le cas où $L=4, u=1$ avec $(x, y, z)=(1,1,1)$. On trouve alors :

$$
\left(x^{\prime}, y, z\right)=(2,1,1), \quad\left(x, y^{\prime}, z\right)=(1,3,1), \quad\left(x, y, z^{\prime}\right)=(1,1,3) .
$$


Si au contraire $y^{\prime}=y$, la configuration correspondante est celle de la figure 5 .

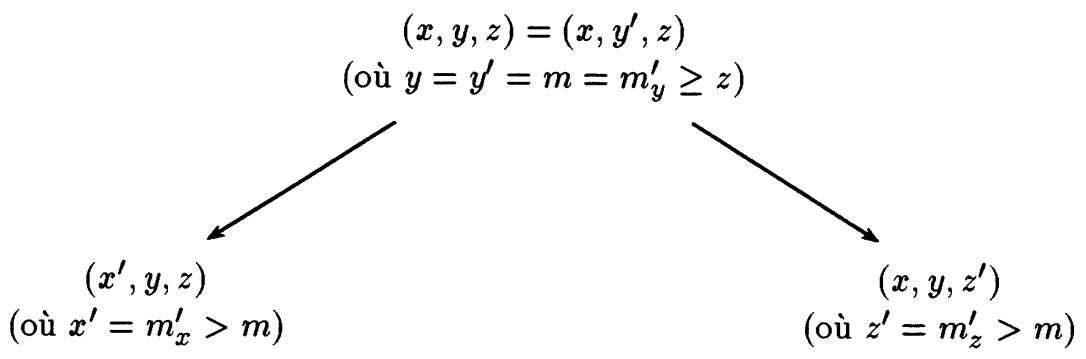

Fig. 5

Dans ce cas, le groupe $G$ n'agit pas transitivement sur l'ensemble $S$ de l'équation (7).

Cas 3. - On suppose $x^{\prime}<x$. Alors $x=m>m_{x}^{\prime}$.

On a aussi, avec l'hypothèse faite sur $L$ :

$$
\begin{aligned}
& y^{\prime}=m_{y}^{\prime}>x=m>y \\
& z^{\prime}=m_{z}^{\prime}>x=m>z .
\end{aligned}
$$

Dans ce cas, la solution $(x, y, z)$ est nécessairement non fondamentale. La configuration correspondante est celle donnée par figure 6 .

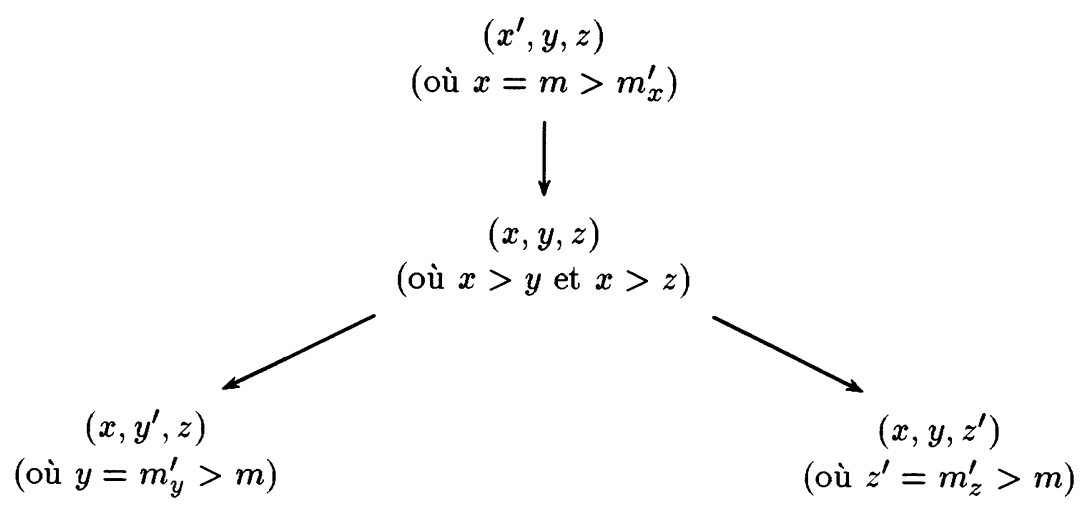

Fig. 6 


\subsubsection{Combinaison des cas précédents}

On combine les différents cas que l'on vient de voir. Toute solution de l'équation diophantienne permet de construire pas à pas, en veillant à faire décroître le nombre $m=\max (x, y, z)>0$, une solution fondamentale. En effet, toute valeur minimale de $m$ correspond à une solution fondamentale par ce qui précède. Cette solution fondamentale permet de construire en sens inverse un arbre infini de solutions contenant celle dont on est parti. Ceci termine la démonstration du théorème 1 .

\subsection{Démonstration du théorème 2}

Le problème est ici de déterminer le nombre de solutions fondamentales que l'on est susceptible de trouver par la méthode précédente. Pour une solution fondamentale $(x, y, z)$, seuls les cas 1 et 2 sont à considérer. On peut donc supposer $x^{\prime} \geq x$ et, comme vu avant, $y \geq z$.

\subsubsection{Cas 2 a), $x^{\prime}>x$ et $y \geq x$}

L'équation s'écrit

$$
f(y)=y^{2}-(L x z) y+\left(x^{2}+z^{2}+u x\right)=0 .
$$

Avec ce qui précède, $y \leq y^{\prime}$. De sorte que $z$ n'étant pas situé entre les deux racines $y$ et $y^{\prime}$ du trinôme $f(y)$, on a :

$$
0 \leq f(z)=z^{2}(2-L x)+x^{2}+u x .
$$

On trouve

- si $x \leq z:(L x-3) z \leq u$; et l'on conclut facilement en distinguant les deux cas $L x \leq 3$ et $L x>3$;

- si $z<x$ :

$$
0 \leq f(x) \leq x^{2}(2-L z)+\left(\frac{3}{L}+u\right) x+\frac{u}{L}
$$

on conclut facilement en distinguant les deux cas $L z>2$ et $L z \leq 2$.

3.2.2 Cas 2 b): $x^{\prime}>x>y \geq z$

L'équation s'écrit

$$
g(x)=x^{2}+(u-L y z) x+y^{2}+z^{2}=0 .
$$


On a, puisque $y$ n'est pas situé entre les deux racines du trinôme :

$$
y(L z-3) \leq u .
$$

On conclut facilement en distinguant les deux cas $L z \leq 3$ et $L z>3$.

3.2.3 Cas $1: x=x^{\prime}>\max (y, z)$

L'équation étudiée s'écrit

$$
h(y)=y^{2}\left(2-\frac{L^{2}}{2} z^{2}\right)+(u L z) y+\left(2 z^{2}-\frac{u^{2}}{2}\right)=0 .
$$

On conclut facilement en distinguant les cas $L z=2$ et $L z>2$. Au total, on ne trouve ainsi qu'un nombre fini de solutions fondamentales. Ceci termine la démonstration du théorème 2 .

\section{Démonstration du théorème 3 et application}

\subsection{La démonstration}

La démonstration du théorème $1 \mathrm{a}$ permis de distinguer différents cas pour les solutions fondamentales. Dans tous les cas, on vérifie que l'on a :

$$
y^{\prime}=L x z-y \geq y
$$

Si l'on pose

$$
2 y=L x z-v \quad(v \in \mathbb{N}),
$$

on peut considérer le nombre entier positif $T$ qui s'écrit facilement sous les deux formes suivantes :

$$
T=(L x z)^{2}+u^{2}=(2 z)^{2}+(2 x+u)^{2}+v^{2} .
$$

Ainsi, la recherche d'une solution fondamentale se fait parmi celles des nombres entiers $T$ décomposables en somme de trois carrés et en somme de deux carrés. Par des résultats classiques (cf. [14, théorèmes 1 et 2, pp. 24-25]) un tel nombre $T$ n'est pas de forme $4^{k}(8 m+7)$. Il est aussi tel que tout diviseur premier $q$ de $T$ congru à 3 modulo 4 apparaisse dans sa décomposition en facteurs premiers avec une puissance paire. 


\section{Serge Perrine}

Inversement, en énumérant ces nombres entiers positifs $T$ possédant deux telles décompositions ( $\mathrm{T}$ ) et en calculant les valeurs $L, u, x, z$ puis $y=(L x z-v) / 2$, on fabrique des équations diophantiennes (7) et des solutions correspondantes. Ces solutions peuvent être fondamentales ou non.

Mais il est facile, avec les définitions données auparavant, de sélectionner les solutions fondamentales. De plus, toute solution fondamentale est ainsi obtenue et ceci donne le théorème 3 .

On présente ici quelques exemples d'équations et de solutions (non nécessairement fondamentales) obtenues par la méthode précédente.

\section{Exemples}

- Avec $T=17=(4 \cdot 1 \cdot 1)^{2}+1^{2}=(2 \cdot 1)^{2}+(2 \cdot 1+1)^{2}+2^{2}$, on trouve la solution fondamentale du cas 2 a) :

$$
(x, y, z)=(1,1,1)
$$

de l'équation $x^{2}+y^{2}+z^{2}=4 x y z-x$.

- Avec $T=65=(4 \cdot 2 \cdot 1)^{2}+1^{2}=(2 \cdot 1)^{2}+(2 \cdot 1+1)^{2}+6^{2}$, on trouve la solution non fondamentale :

$$
(x, y, z)=(2,1,1)
$$

de l'équation $x^{2}+y^{2}+z^{2}=4 x y z-x$.

- Avec $T=12241=(2 \cdot 8 \cdot 6)^{2}+55^{2}=(2 \cdot 6)^{2}+(2 \cdot 8+55)^{2}+84^{2}$, on trouve la solution fondamentale du cas $2 \mathrm{~b}$ ) :

$$
(x, y, z)=(8,6,6)
$$

de l'équation $x^{2}+y^{2}+z^{2}=2 x y z-55 x$.

- Avec $T=1096=(2 \cdot 5 \cdot 3)^{2}+14^{2}=(2 \cdot 3)^{2}+(2 \cdot 5+14)^{2}+22^{2}$, on trouve la solution fondamentale du cas 1 :

$$
(x, y, z)=(5,4,3)
$$

de l'équation $x^{2}+y^{2}+z^{2}=2 x y z-14 x$.

(Cette solution est également fondamentale du cas 1 pour l'équation $x^{2}+y^{2}+z^{2}=3 x y z-26 x$ et pour la décomposition $T=2701=$ $\left.(3 \cdot 5 \cdot 3)^{2}+26^{2}=(2 \cdot 3)^{2}+(2 \cdot 5+26)^{2}+37^{2}.\right)$ 


\subsection{Application à la recherche des solutions fondamentales}

En décrivant par ordre croissant l'ensemble des nombres entiers positifs $T \in \mathbb{N}^{*}$ et pour chacun de ces nombres, en recherchant toutes les décompositions nécessairement en nombre fini de forme :

$$
T=A^{2}+B^{2}=C^{2}+D^{2}+E^{2}
$$

où $A \in \mathbb{N}^{*}, B \in \mathbb{N}^{*}, C \in \mathbb{N}^{*}, D \in \mathbb{N}^{*}$ et $E \in \mathbb{N}$.

En posant ensuite successivement :

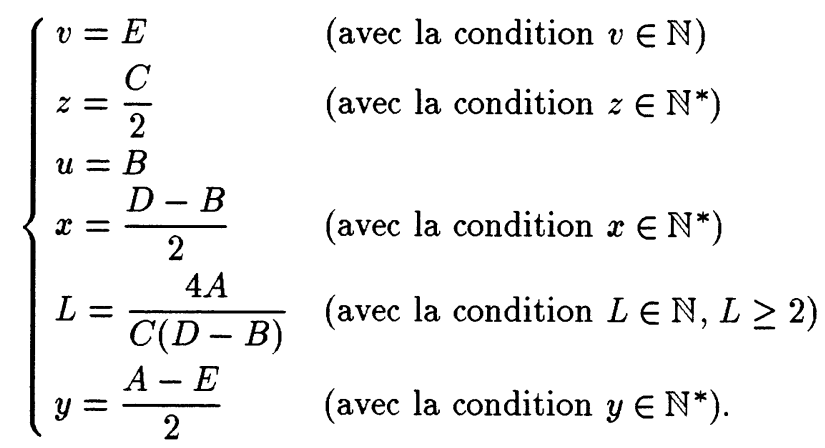

En s'assurant enfin que les conditions nécessaires sont vérifiées, on détermine toutes les équations diophantiennes (7) possédant une infinité de solutions. On peut ne conserver que les solutions fondamentales en testant des inégalités évidentes.

La procédure est facilement automatisable. Elle a permis à l'auteur du présent article de constituer une table assez importante d'équations diophantiennes (7) possédant des solutions, et de leurs solutions fondamentales.

On a donné, ci-dessus, un extrait de cette table pour $L=3$ et $u \leq 20$. La procédure permet de retrouver pour $L=4$ et $u \leq 25$ les solutions fondamentales données dans [12]. Cusick a calculé ces dernières par une méthode déduite de celle de Herzberg [15] également transposable à l'équation (7) pour démontrer le théorème 2 .

\section{Remarques finales}

Remarque 5.1. - On peut aisément généraliser les méthodes qui précèdent aux équations $\left(5^{\prime}\right)$, en oubliant la condition restrictive imposée dans [10] ou [17] :

$$
L=3+a_{12}+a_{23}+a_{31}+u_{1}+u_{2}+u_{3} .
$$


Pour cela, les transformations $\phi_{1}, \phi_{2}$ et $\phi_{3}$ à considérer sont données par

$$
\begin{aligned}
& x^{\prime}=L y z-x-a_{12} y-a_{31} z-u_{1} \\
& y^{\prime}=L x z-y-a_{12} x-a_{23} z-u_{2} \\
& z^{\prime}=L x y-z-a_{31} x-a_{23} y-u_{3} .
\end{aligned}
$$

Elles engendrent un groupe $G$ produit libre de trois copies du groupe à deux éléments $\mathbb{Z} \backslash 2 \mathbb{Z}$. Le groupe $G$ agit sur l'ensemble $S$ des solutions de l'équation considérée. Il découpe $S$ en orbites disjointes et chaque orbite peut être caractérisée par l'un de ses éléments : la solution fondamentale correspondante.

Ainsi toutes les solutions de l'équation ici considérée sont données à partir d'un ensemble de solutions fondamentales. Il reste à caractériser les équations du type ici considéré dont l'ensemble de solutions fondamentales n'est pas fini. L'auteur conjecture qu'il n'en existe pas.

Remarque 5.2. - Les méthodes qui précèdent permettent sans difficulté de calculer tous les points à coordonnées entières des surfaces réelles d'équations affines :

$$
x^{2}+y^{2}+z^{2}=L x y z-a_{12} x y-a_{23} y z-a_{31} x z-u_{1} x-u_{2} y-u_{3} z
$$

\section{Bibliographie}

[1] Cassels (J. W. S.) .- An introduction to diophantine approximation, Cambridge Tracts in Mathematics 45, Cambridge University Press (1957).

[2] Flahive (M. E.) et Cusick (T. W.) - - The Markoff and Lagrange Spectra, Mathematical Surveys and Monographs 30, American Mathematical Society (1989).

[3] Mordell (L. J.) .- Diophantine equations, Academic Press New-York (1969).

[4] Hurwitz (A.) .- Uber eine Aufgabe der unbestimmten Analysis, Archiv. der Math. und Phys. 3, $\mathrm{n}^{\circ} 14$ (1907), pp. 185-196.

[5] SCHWARTz et MUhLY (H. T.) .- On a class of cubic diophantine equations, J. Lond. Math. Soc. 32 (1957), pp. 379-383.

[6] Mills (W. H.) .- Certain diophantine equations linear in one unknown, Canad. J. Math. 8 (1956), pp. 5-12.

[7] Pollington (A. D.) et Moran (W.) .- Number Theory with an emphasis on the Markoff spectrum, M. Dekker New-York (1993). 
Sur des équations diophantiennes généralisant celle de Markoff

[8] Perrine (S.) .- Approximation diophantienne (théorie de Markoff), Thèse présentée à l'Université de Metz (décembre 1988).

[9] Silverman (J. H.) .- The Markoff equation $X^{2}+Y^{2}+Z^{2}=a X Y Z$ over quadratic imaginary fields, J. Number Theory 35, tome 1 (1990), pp. 72-104.

[10] MANin (Y.) et TSFASMAN (M. A.) .- Rational varieties : algebra, geometry and arithmetic, Uspekhi Mat. Nauk 41, $\mathrm{n}^{\circ} 2$ (1986), pp. 43-94; Russian math. surveys 41 (1986) pp. 51-116.

[11] Perrine (S.) .- Sur une généralisation de la théorie de Markoff, J. Number Theory 37 (1991), pp. 211-230.

[12] CUSICK (T. W) .- On Perrine's generalized Markoff equations, Aequationes Mathematicae 46, $\mathrm{n}^{\circ} 3$ (1993), pp. 203-211.

[13] Frobenius (G.) .- Uber die Markoffschen Zahlen, Preussiche Akad. Wiss. SB (1913), pp. 458-487.

[14] Grosswald (E.) .- Representations of integers as sums of squares, Springer Verlag, New-York - Berlin (1985).

[15] Herzberg (W.) .- On a problem of Hurwitz, Pacific J. Math. 50 (1974), pp. 485-493.

[16] CoHn (H.) .- Representation of Markoff's binary quadratic forms by geodesics on a perforated torus, Acta Arithmetica XVII (1971), pp. 123-136.

[17] El Khuti el'Huti (M. Kн.) .- Cubic surfaces of Markov type, Mat. Sb 93 (1974), pp. 331-336; Math USSR-Sb 22 (1974), pp. 333-348.

[18] BARAgAR (A.) . - Integral Solutions of Markoff Hurwitz equations, J. Number Theory 49 (1994), pp. 27-44. 